\title{
Prospects to regain the ability to accommodate
}

\author{
Henk A. Weeber ${ }^{1}$
}

Received: 11 December 2015 / Accepted: 17 December 2015 / Published online: 18 February 2016

(C) Springer-Verlag Berlin Heidelberg 2016

Over one billion people around the world are affected by presbyopia [1]. A major factor causing presbyopia is stiffening of the crystalline lens. Elasticity, as a material property of the crystalline lens matrix, is often expressed by the Young's modulus, a unit for stiffness named after Thomas Young. Interestingly, Thomas Young also studied accommodation and developed an instrument to measure objectively the accommodative amplitude. In 1801 he concluded that "in general, I have reason to think that the faculty diminishes to some degree as persons advance in life" [2]. This phenomenon has been confirmed in later years by measuring the accommodative amplitude of thousands of subjects, and it was soon suggested that the decline in accommodative amplitude with age should be attributed to stiffening of the crystalline lens [3].

Traditionally, presbyopia is treated with reading spectacles or contact lenses, using bifocal contact lenses or some sort of monovision. More recently, laser refractive surgery has gained popularity as the procedure has options of using multifocality and monovision for treating presbyopia. Another and more invasive alternative is to perform clear lens extraction, in which the natural crystalline lens is replaced by a multifocal or accommodating intraocular lens or a by lens that extends the range of vision. Scleral approaches are also being explored [4]. A less invasive way to combat presbyopia, proposed in 1998 by Myers and Krueger, is to alter the properties of the crystalline lens by photoablation or photodisruption in the crystalline lens by a laser [5]. While mechanical changes in the lens material as a result of laser treatment were already

Henk A. Weeber

henk.weeber@abbott.com

1 AMO Groningen b.v., Van Swietenlaan 5, 9728

NX Groningen, Netherlands foreseen [6], Krueger and co-workers demonstrated a change in pliability of the lens as a whole, suggesting that presbyopia may be alleviated or reversed [7]. So far, only moderate changes in pliability have been observed. A factor influencing the effect of the procedure is the geometrical pattern inside the lens that is treated by the laser. Studies have concentrated on cutting inside the lens, generating gliding planes along which internal deformations can take place [8].

The search for the optimal gliding planes that maximize lens pliability is the subject of the paper "Finite element modelling of radial lentotomy cuts to improve the accommodation performance of the human lens" by Burd \& Wilde in the current issue of in Graefe's Archive for Clinical and Experimental Ophthalmology. Instead of experimental studies, e.g. using cadaver eyes, the authors use computational methods to compare the effects of different cutting geometries. Computational methods avoid issues with measurement uncertainties, as well as variance between test specimen and post mortem degradation of cadaver lenses. On the down side, the validity of computational outcomes depends heavily on the validity of the input parameters of the model: geometry, material properties, and boundary conditions. It has been well demonstrated by the same authors in previous studies that the way that the behavior of ocular tissues is modeled has a significant effect on the outcomes $[9,10]$. In the current paper the authors demonstrate this once again, by modeling the behavior of the lens matrix in two different ways. First, they model the lens matrix as an incompressible material that is initially linear elastic, but becomes non-linear due to large deformations. This approach of modelling the mechanical behavior of the crystalline lens is most often used in current literature. Then, as an alternative, they introduce a poroelastic material model, which represents the lens matrix as a two-phase mixture of an incompressible fluid and a compressible hyperelastic solid matrix. Whether this is a more realistic 
representation of the crystalline lens mechanics is unknown, but intuitively this should at least better model the area of the gliding planes. Only when modeling the lens as a poroelastic material some useful change in lens power was achieved. The results of the study indicate that the obtained gain in accommodation by this treatment is rather small, and as the authors state, the study presents a rather pessimistic assessment of the likely effectiveness of lentotomy procedures based on radial cuts alone. Nevertheless, experimental studies are still ongoing, and many of the biological impacts of the laser cuts remain to be investigated, all of which may influence the reallife results.

\section{References}

1. Holden BA et al (2008) Global vision impairment due to uncorrected presbyopia. Arch Ophthalmol 126(12):1731-1739

2. Young T (1801) On the Mechanism of the Eye. Philisophical Trans 92:P52
3. Donders FC (1864) On the anomalies of accommodation and refraction of the eye. New Sydenham Soc, London, p 205

4. Shiple DA, Aquavella J (2014) A review of surgical advancements for the correction of presbyopia. Expert Rev Ophthalmol 9(1):4348

5. Myers RI, Krueger RR (1998) Novel approaches to correction of presbyopia with laser modification of the crystalline lens. J Refract Surg 14(2):136-139

6. Gwon A et al (1995) Focal laser photophacoablation of normal and cataractous lenses in rabbits: preliminary report. J Cataract Refract Surg 21(3):282-286

7. Krueger RR, Xiaoqi KS, Stroh J, Myers R (1999) Experimental increase in accommodative potential following Nd:YAG laser photodisruption of paired cadaver lenses. ARVO abstract 1535 . Invest Ophthalmol Vis Sci (Fort Lauderdale, FL.)

8. Ripken T, Oberheide U, Fromm M, Schumacher S, Gerten G, Lubatschowski H (2008) fs-Laser induced elasticity changes to improve presbyopic lens accommodation. Graefes Arch Clin Exp Ophthalmol 246(6):897-906

9. Burd HJ, Judge SJ, Flavell MJ (1999) Mechanics of accommodation of the human eye. Vis Res 39(9):1591-1595

10. Burd HJ, Wilde GS, Judge SJ (2006) Can reliable values of Young's modulus be deduced from Fisher's (1971) spinning lens measurements? Vision Res, (8-9), 1346-1360 